\title{
Controllability measures for disturbance rejection
}

\author{
SIGURD SKOGESTAD $*+$ and ERIK A. WOLFF $\dagger$
}

Keywords: Input-output controllability, control structure design, relative gain array, relative disturbance gain

Some plants have better "built-in" disturbance rejection capabilities than others, that is, their dynamic resilience (controllability) with respect to disturbance rejection is better. In the paper we consider controller independent disturbance measures for six classes of problems:

1. Open-loop disturbance sensitivity.

2. Disturbance sensitivity for decentralized control.

3. Disturbance sensitivity for system under partial control.

4. Input magnitude for rejecting disturbances.

5. Output magnitude for disturbances in the presence of input limitations.

6. Maximum disturbance range.

For all six problems we obtain frequency-dependent measures, and appropriate scaling of the variables is crucial for interpreting these measures. In the paper we also discuss the relationship between these measures and the Relative Disturbance Gain (RDG) of McAvoy and coworkers and the disturbance condition number of Skogestad and Morari.

\section{Introduction}

The objective of this paper is to consider "controllability" measures for disturbances (often called "loads"). By the input-output "controllability" or "dynamic resilience" of a plant we mean the inherent control properties of the plant. That is, if a plant has poor controllability, then the responses for that plant will be poor no matter what controller we select to use.

Notation. In this paper we consider linear transfer function models on the form

$$
y(s)=G(s) u(s)+G_{d}(s) d(s)
$$

where $u$ is the vector of manipulated inputs, $d$ is the vector of (physical) disturbances, and $y$ is the vector of outputs (controlled variables). The objective is to keep the error $e=y-r$ small, where $r$ is the vector of reference signals (setpoints). $G(s)$ and $G_{d}(s)$ are transfer function matrices which need not be square. In many cases we consider a single disturbance at the time and $d$ is a scalar, and in this case we write $g_{d}$ instead of $G_{d}$ to show explicitly that it is a vector. Throughout the paper subscript $i$ denotes a particular output, subscript $j$ denotes a particular input, subscript $k$ (or no subscript) denotes a particular disturbance, while subscript $l$ denotes a set of variables. The linear feedback controller is denoted $C(s)$. In the paper we refer to the loop transfer

Received 12 March 1996.

*E-mail: SKOGE@KJEMI.NTNU.NO, phone: 47-73594154, fax: 47-73594080.

†Chemical Engineering, University of Trondheim-NTH, N-7034 Trondheim, Norway.

This paper was presented at the IFAC Workshop on Interactions between Process Design and Process Control, London, Sept. 6-8, 1992.

Reprinted with kind permission from Pergamon Press. 
function $L=G C$, the sensitivity $S=(I+L)^{-1}$, and the complimentary sensitivity $T=L(I+L)^{-1}$. We define the closed-loop bandwidth, $\omega_{B}$, as the lowest frequency where $\sigma(L(j \omega))=1$, where $\underline{\sigma}$ is the minimum singular value. The Laplace variable $s$ or frequency argument $s=j \omega$ is usually omitted to simplify notation.

Surprisingly, the issue of disturbances has not been widely discussed in the general literature on controllability analysis. Of course, it has been known for a long time that disturbance rejection is an important property of the plant, and this issue has been discussed in detail for distillation columns (e.g. McCune and Gallier 1973, Waller et al. 1988, Skogestad and Morari 1987b). However, there has been a lack of systematic tools for quantifying the effect of disturbances. Morari (1983) considers the magnitude of the inputs needed for disturbance rejection, and argues that the minimum singular value of the plant, $\underline{\sigma}(G)$, may provide a useful measure. However, he does not use any information about the disturbance model, $g_{d}(s)$. Stanley $e t$ al. (1985) introduced a dimensionless measure for disturbance which they called the Relative Disturbance Gain (RDG). Morari et al. (1985) consider the allowed magnitude of disturbances to achieve feasible steady-state operation, and denote their measure the Resiliency Index (RI). Shimizu and Matsubara (1985) discuss the direction of combined disturbances in the frequency domain using the singular value decomposition. Skogestad and Morari (1987a) present a similar analysis, but also consider the direction of an individual disturbance. They stress that in multivariable systems some disturbances may be difficult to reject if they are in the "bad" direction compared to the direction of the plant, and to quantify this they introduce the disturbance condition number, $\gamma_{d}(G)$. They also provide a performance interpretation of the RDG and extend it to non-zero frequencies. Perkins (1989) also considers frequency-dependent disturbances and uses the magnitude of the disturbance transfer function, $\left|g_{d}\right|$ or $\bar{\sigma}\left(G_{d}\right)$, as a measure of the expected output error in the absence of control. Luyben (1988) stresses that the choice of the control structure may strongly influence the sensitivity to disturbances. Skogestad and Hovd (1990) and Hovd and Skogestad (1992) argue that for decentralized control one should use the Closed-loop disturbance gain (CLDG) rather than $G_{d}$ when evaluating the effect of disturbances.

The main topic of the paper is to present controller-independent measures for evaluating a plant's sensitivity to disturbances. In all cases we consider frequencydependent measures, and the class of disturbances we shall consider is sinusoids $d(j \omega)$ with magnitude less than 1 at each frequency $\omega$.

\section{Preliminaries}

Vector norms. In this paper we will at each frequency study the magnitude of $d$, $u$ and $y$. In most cases we will consider a single disturbance at a time and assume $d$ is a scalar, and its magnitude is then simply $|d(j \omega)|$, which is the absolute value of the complex number $d(j \omega)$. To evaluate the magnitude of the input vector $u$ and output vector $y$ we shall use either vector 2-norm or the vector infinity-norm, which for a (complex) vector $y=\left(y_{1}, y_{2}, \ldots, y_{n}\right)^{T}$ at a given frequency are defined as

$$
\begin{aligned}
\|y\|_{2} & =\sqrt{y_{1}^{2}+y_{2}^{2}+\ldots+y_{n}^{2}} \\
\|y\|_{\infty} & =\max _{i}\left|y_{i}\right|
\end{aligned}
$$


Singular values. These are directly related to the vector 2-norm. Specifically, we have for $y=A x$ that

$$
\underline{\sigma}(A) \leq \frac{\|y\|_{2}}{\|x\|_{2}} \leq \bar{\sigma}(A)
$$

where $\bar{\sigma}(A)$ is the maximum singular value, and $\underline{\sigma}(A)$ the minimum singular value of $A$. We may choose the directions for $x$ such that either the lower or upper bounds in (4) is an equality.

Feedback control. When feedback control is used, $u=C(s)(r-y)$, the closed-loop response to a setpoint $r$ and a disturbance $d$ becomes

$$
e=y-r=-S r+S G_{d} d ; \quad u=C S r-C S G_{d} d
$$

The objective of this paper is to study the inherent control limitations of the plant, and we need measures that are independent of the controller. It is then clarifying to study the special case of perfect control (Morari, 1983). From Eq. (1) we see that for a square plant perfect control $(y=r)$ is achieved if we apply the following input signals

$$
u=G^{-1} r-G^{-1} G_{d} d
$$

In practice we may achieve something close to this by applying feedback control: since $T=G C S$, Eq. (5) yields

$$
u=G^{-1} T r-G^{-1} T G_{d} \mathrm{~d}
$$

At frequencies lower than the bandwidth $\omega_{B}$ the following approximation holds ${ }^{1}$

$$
S \approx L^{-1} ; T \approx I ; \omega \leq \omega_{B}
$$

and we get

$$
u(j \omega) \approx G^{-1} r(j \omega)-G^{-1} G_{d} d(j \omega) ; \omega \leq \omega_{B}
$$

which is identical to (6). Note that (9) is independent of the controller, while the corresponding expression (5) for the control error is not:

$$
e(j \omega)=y-r \approx-L^{-1} r+L^{-1} G_{d} d ; \omega \leq \omega_{B}
$$

\section{Existing controllability measures}

$\boldsymbol{R G A}$. The most common controllability measure is probably the RGA which was first introduced by Bristol (1966). For a square plant $G$ the RGA is defined as the ratio of the "open-loop" and "closed-loop" gains between input $j$ and output $i$

$$
\lambda_{i j}(s)=\frac{\left(\partial y_{i} / \partial u_{j}\right)_{u_{l \neq j}}}{\left(\partial y_{i} / \partial u_{j}\right)_{y_{l \neq i}}}=g_{i j}(s)\left[G^{-1}(s)\right]_{j i}
$$

(Proof: see Appendix). An RGA matrix can be computed at any frequency using the formula

$$
\Lambda(s)=G(s) \times\left(G^{-1}(s)\right)^{T}
$$

where the $\times$ symbol denotes element by element multiplication (Hadamard or Schur product). One important advantage of the RGA is that it is independent of input and output scaling.

\footnotetext{
'In cases where some loops are not closed we will have $\sigma(L)=0$, and the approximation $S \approx L^{-1}$ does not hold, but in such cases similar approximations may be applied to subsystems.
} 
PRGA. One inadequacy of the RGA (e.g. McAvoy 1983, p. 166) is that it may indicate that interaction is no problem, but significant one-way coupling may exist. This follows since the $\Lambda=I$ when $G(s)$ is triangular. To overcome this problem Hovd and Skogestad (1992) introduce the performance relative gain array (PRGA) The PRGA-matrix is defined as

$$
\Gamma(s)=\tilde{G}(s) G(s)^{-1}
$$

where $\tilde{G}(s)$ is the matrix consisting of only the diagonal elements of $G(s)$, i.e. $\tilde{G}=\operatorname{diag}\left\{g_{i i}\right\}$. The elements of $\Gamma$ are given by

$$
\gamma_{i j}(s)=g_{i i}(s)\left[G^{-1}(s)\right]_{i j}=\frac{g_{i i}(s)}{g_{j i}(s)} \lambda_{j i}(s)
$$

Note that the diagonal elements of RGA and PRGA are identical, but otherwise PRGA does not have all the nice algebraic properties of the RGA.

Condition number. The condition number, $\gamma(G)=\bar{\sigma}(G) / \underline{\sigma}(G)$, is also often used as a controllability measure, and a plant with a large condition number is called ill-conditioned.

\section{Disturbance measures}

RDG. In the spirit of the RGA, Stanley et al. (1985) introduced the Relative Disturbance Gain (RDG) as the ratio between the input $u_{j}$ needed when rejecting a disturbance $k$ in all outputs and the input $u_{j}$ needed when rejecting the disturbance $k$ in only one output $j$. Skogestad and Morari (1987a) gave this measure a performance interpretation and found that it may be evaluated at any frequency using (see Appendix)

$$
\beta_{j k}=g_{j j}\left[G^{-1} G_{d}\right]_{j k} / g_{d j k}=\left[\tilde{G} G^{-1} G_{d}\right]_{j k} / g_{d j k}
$$

CLDG. Skogestad and Hovd (1990) introduced a measure that is closely related to both the RDG and PRGA. For a disturbance $k$ and an output $i$, the Closed-loop Disturbance Gain (CLDG) is defined by

$$
\delta_{i k}=g_{i i}\left[G^{-1} G_{d}\right]_{i k}=\left[\tilde{G} G^{-1} G_{d}\right]_{i k}=\left[\Gamma G_{d}\right]_{i k}
$$

where $\Gamma$ is the PRGA. The reason for the name CLDG will become clear later. The CLDG is closely related to the RDG since from (15)

$$
\beta_{i k}(s)=\delta_{i k}(s) / g_{d i k}(s)
$$

Disturbance condition number. To study specifically the direction of a disturbance, Skogestad and Morari (1987a) introduced the disturbance condition number of the matrix $A$ ( $A$ may be $G$ or $L=G C$ )

$$
\gamma_{d}(A)=\frac{\left\|A^{-1} g_{d}\right\|_{2}}{\left\|g_{d}\right\|_{2}} \bar{\sigma}(A)
$$

\section{Scaling}

All interpretations and examples in this paper assume that appropriate scaling has been performed. The RGA has the advantage of being scaling independent, but for the other measures it is crucial that the variables are scaled properly. In general, the variables should be scaled to be within the interval -1 to 1 , that is, their desired or 
expected magnitudes should be normalized to be less than 1 at each frequency. Recommended scalings:

- Inputs $(u)$ : Normalize $u_{j}$ with respect to its allowed range.

- Outputs $(y)$ : Normalize $e_{i}$ with respect to its allowed range.

- Disturbances $(d)$ : Normalize $d_{k}$ with respect to its expected range.

The measures depend on scaling as follows:

- RGA: independent of scaling

- PRGA: depends on scaling of $y$ (except diagonal elements)

- $G_{d}$, CLDG, RDG: depend on scaling of $d$ and $y$

- $\gamma(G), \gamma_{d}(G)$ : depend on scaling of $u$ and $y$.

Comment: In this paper we scale directly the transfer matrices $G$ and $G_{d}$ and assume that the expected or allowed magnitude of the signals $d, u, e$ and $r$ does not vary with frequency. If their magnitudes vary then we should rather scale the signals using frequency-dependent weights. This signal approach is also more general, for example, if the setpoints do not have same size as the allowed errors (as we implicitly have to assume).

\section{Results on disturbance measures}

The main objective of this paper is to derive simple controller-independent measures, and we shall consider disturbance measures for six different problems.

\subsection{Interpretation of open-loop disturbance sensitivity, $\mathrm{G}_{\mathrm{d}}$}

SISO plants. Select at each frequency $d(j \omega)=1$ (the worst case). Then the output at this frequency is $y(j \omega)=g_{d}(j \omega) d=g_{d}(j \omega)$. Now, note that the output has been scaled such that we require at each frequency $|y(j \omega)|<1$. Consequently, at frequencies where $\left|g_{d}\right|$ is larger (larger than 1), we need control (feedforward or feedback) in order to reject this disturbance. Typically, $\left|g_{d}\right|$ is larger than 1 at low frequencies and drops to zero at high frequencies. The frequency, $\omega_{d}$, where $\left|g_{d}(j \omega)\right|$ crosses 1 is then a useful controllability measure, since it yields the minimum bandwidth requirement for feedback control. Plants with a small value of $\left|g_{d}\right|$ and $\omega_{d}$ are preferable (better dynamic resilience) since the need for control is less, or alternatively, with a given controller the effect of the disturbance on the output is less.

Example. Consider the disturbance model

$$
g_{d}(s)=k_{d} /\left(1+\tau_{d} s\right)
$$

where $k_{d}=10$ and $\tau_{d}=2$ [min]. Scaling has been applied to $g_{d}$, so this means that with no control, the effect of a disturbance on the output at low frequencies is $k_{d}=10$ times larger than what we allow. Thus control is required, and since $g_{d}$ crosses 1 at a frequency $\omega_{d} \approx k_{d} / \tau_{d}=0.5 \mathrm{rad} / \mathrm{min}$, the minimum bandwidth requirement for disturbance rejection using feedback control is $\omega_{B}>0.5 \mathrm{rad} / \mathrm{min}$. Waller et al. (1988) found that the disturbance parameter $k_{d} / \tau_{d}$ correlated well with the observed disturbance sensitivity for various distillation control configurations.

Use of feedback control. In this case the response to the disturbance is $y(s)=S(s) g_{d}(s) d(s)$, and at a given frequency where $d(j \omega)=1$, the requirement of $|y(j \omega)|<1$ is satisfied

$$
\text { if and only if }|S(j \omega)|<1 /\left|g_{d}(j \omega)\right|
$$


At low frequencies, $\omega \leq \omega_{B}$, where feedback is effective and we have $|L(j \omega)|>1$ and $S(j \omega) \approx 1 / L(j \omega),(20)$ becomes

$$
|y(j \omega)|<1 \text { if and only if }|L(j \omega)|>\left|g_{d}(j \omega)\right|, \omega \leq \omega_{B}
$$

Thus, at frequencies where feedback is needed for disturbance rejection $\left(\left|g_{d}\right|>1\right)$, we must require the loop transfer function $|L(j \omega)|$ to be larger than the disturbance transfer function $\left|g_{d}(j \omega)\right|$ (appropriately scaled).

MIMO plants. Consider one disturbance and select at each frequency $d(j \omega)=1$ (the worst-case disturbance). We then have $y=S g_{d} .1$ and from (4)

$$
\underline{\sigma}(S)\left\|g_{d}\right\|_{2} \leq\|y\|_{2} \leq \bar{\sigma}(S)\left\|g_{d}\right\|_{2}
$$

At low frequencies we have $S \approx L^{-1}$ and $\underline{\sigma}(S) \approx \underline{\sigma}\left(L^{-1}\right)=1 / \bar{\sigma}(L), \bar{\sigma}(S) \approx 1 / \underline{\sigma}(L)$, and we derive

$$
\frac{\left\|g_{d}\right\|_{2}}{\bar{\sigma}(L)} \leq\|y\|_{2} \leq \frac{\left\|g_{d}\right\|_{2}}{\underline{\sigma}(L)}, \omega \leq \omega_{B}
$$

which gives

$$
\begin{gathered}
\|y\|_{2}<1 \text { only if } \bar{\sigma}(L)>\left\|g_{d}\right\|_{2}, \omega \leq \omega_{B} \\
\|y\|_{2}<1 \text { if } \underline{\sigma}(L)>\left\|g_{d}\right\|_{2}, \omega \leq \omega_{B}
\end{gathered}
$$

For MIMO plants we have a bandwidth region ranging from $\omega_{B}$ (where $\underline{\sigma}(L)$ crosses 1) to $\omega_{B}^{\prime}$ (where $\bar{\sigma}(L)$ crosses 1 ). For ill-conditioned systems the difference between the bandwidth in the worst direction $\left(\omega_{B}\right)$ and in the best direction $\left(\omega_{B}^{\prime}\right)$ may be large. For a single disturbance consider the frequency $\omega_{d}$ where $\left\|g_{d}\right\|_{2}$ crosses 1 . Then we must require that $\omega_{B}^{\prime}>\omega_{d}$ and we may have to require $\omega_{B}>\omega_{d}$ (depending on the direction of the disturbance).

\subsection{Disturbance sensitivity for decentralized control}

For SISO systems we found at low frequencies that the loop transfer function $L=g c$ must be larger than $g_{d}$ in magnitude to achieve disturbance rejection. Decentralized control is frequently used for MIMO plants, and we would like to derive for this case similar bounds on each loop transfer functions, $L_{i}=g_{i i} c_{i}$. To this end, assume that $G$ is a square matrix, and $C(s)$ is diagonal with entries $c_{i}(s)$.

Eq. (10) applies at low frequencies where $\underline{\sigma}(L)>1$. For $g_{i i} \neq 0$, we may write $L=G C=G(\tilde{G})^{-1} \tilde{G} C$ and (10) may then be written as

$$
e=y-r \approx-(\tilde{L})^{-1} \tilde{G} G^{-1} r+(\tilde{L})^{-1} \tilde{G} G^{-1} G_{d} d, \omega \leq \omega_{B}
$$

where $\tilde{L}$ has as diagonal elements the individual loop gains

$$
\tilde{L}=\tilde{G} C=\operatorname{diag}\left\{L_{i}\right\} ; L_{i}=g_{i i} c_{i}
$$

For a particular disturbance $k$ and a setpoint change $j$, (26) yields for output $i$ (closed-loop response for loop $i$ with all loops closed)

$$
e_{\mathrm{i}} \approx-\frac{\gamma_{i j}}{L_{i}} r_{j}+\frac{\delta_{i k}}{L_{i}} d_{k} ; \omega \leq \omega_{B}
$$

where $\gamma_{i j}=\left[\tilde{G} G^{-1}\right]_{i j}$ is the performance relative gain (PRG), and $\delta_{i k}=\left[\tilde{G} G^{-1} G_{d}\right]_{i k}$ is the closed-loop disturbance gain (CLDG). To get a better interpretation of the RGA and 
CLDG consider the response $\hat{e}_{i}$ to a setpoint change $r_{i}$ and a disturbance $d_{k}$ when only loop $i$ is closed (closed-loop response for loop $i$ with all other loops open)

$$
\hat{e}_{i} \approx-\frac{1}{L_{i}} r_{i}+\frac{g_{d i k}}{L_{i}} d_{k} ; \omega \leq \omega_{B}
$$

(29) applies at low frequencies where $\left|L_{i}\right|>1$. A comparison of equations (29) and (28) shows how the control error $e_{i}$ is changed by closing the other loops. We see that

- For a setpoint change $r_{i}$ the change is given by the relative gain, $\lambda_{i i}=\gamma_{i i}$.

- Similarly, for a disturbance $d_{k}$ the change is given by the relative disturbance gain (RDG), $\beta_{i k}=\delta_{i k} / g_{d i k}($ recall (17)).

- We also note that the open-loop disturbance gain, $g_{d i k}$, is replaced by the closed-loop disturbance gain, $\delta_{i k}$. Thus $\delta_{i k}$ represents for loop $i$ the apparent (open-loop) disturbance gain from disturbance $k$ to output $i$ when all loops are closed.

From (28) we see that to achieve $\left|y_{i}(j \omega)\right|<1$ for a unit disturbance $\left|\delta_{k}(j \omega)\right|=1$, we must require that the loop gain, $\left|L_{i}\right|$, is larger than the CLDG, $\left|\delta_{i k}\right|$ (at lower frequencies where $\left.\left|L_{i}\right|>1\right)$. The frequency where $\left|\delta_{i k}(j \omega)\right|$ crosses 1 gives the minimum bandwidth requirement in loop $i$ for this disturbance. It should be less than the bandwidth that can be achieved in practice, which will be limited by time delays, RHP zeros, etc. A plot of $\left|\delta_{i k}(j \omega)\right|$ will give useful information about which disturbances $k$ are difficult to reject.

\subsection{Disturbance sensitivity for system under partial control}

The open-loop disturbance sensitivity for an output $i$ and disturbance $k$ is

$$
\left(\partial y_{i} / \partial d_{k}\right)_{u}=g_{d i k}
$$

We now want to find the corresponding disturbance sensitivity with all the other outputs $l \neq i$ perfectly controlled. For a square plant the following analytical expression is derived (see Appendix)

$$
P D G_{i j k}=\left(\partial y_{i} / \partial d_{k}\right)_{\mathrm{u}_{\mathrm{j}}, y_{l \neq i}}=\left[G^{-1} G_{d}\right]_{j k} /\left[G^{-1}\right]_{j i}
$$

We denote this measure the partial disturbance gain (PDG). The term partial is used since the system is only partially controlled. Note that we get a different value for each input $u_{j}$ left in manual and thus get an array of PDGs with 3 indices $(i, j$ and $k)$. The PDG is a useful measure for cases where it may be difficult to reject disturbances in all outputs simultaneously, for example for plants with large RGA-elements, and one may want to consider having one output $y_{i}$ uncontrolled. For a particular disturbance, one should then check if there exists a particular pairing of $y_{i}$ and $u_{j}$ for which the PDG is less than 1 in magnitude. For simultaneous disturbances we should evaluate the worst overall effect by taking for each "pairing" the sum of element magnitudes. This gives rise to a combined PDG-matrix, denoted $G_{P D G}$, with elements

$$
\left[G_{P D G}\right]_{i j}=\sum_{k}\left|[P D G]_{i j k}\right|
$$

It is desirable to find "uncontrolled pairings" $u_{j}-y_{i}$ where the $G_{P D G}$-element is less than 1 .

For the case $j=i$ (that is, we have paired up the uncontrolled output with the input 
we want in manual), the PDG is equal to the ratio between the CLDG and the corresponding RGA-element (see Appendix):

$$
\left(\partial y_{i} / \partial d_{k}\right)_{u_{i}, y_{l \neq i}}=\delta_{i k} / \lambda_{i i}
$$

Another measure that may be of interest is the relative change in disturbance sensitivity caused by controlling all the other outputs. This measure, denoted the relative-partial disturbance gain (RPDG) is defined as the ratio between (31) and (30). For the case $i=j$ the RPDG is equal to the ratio between the RDG and the RGA

$$
\operatorname{RPDG}(i=j): \frac{\left(\partial y_{i} / \partial d_{k}\right)_{u_{i}, y_{l \neq 1}}}{\left(\partial y_{i} / \partial d_{k}\right)_{u}}=\frac{\delta_{i k}}{\lambda_{i i} g_{d i k}}=\frac{\beta_{i k}}{\lambda_{i i}}
$$

Note that both the RDG and the RPDG yield for an output $i$ and a disturbance $k$ the change in disturbance sensitivity caused by controlling all the other outputs perfectly. The difference is that the PRDG $\left(=\beta_{i k} / \lambda_{i i}\right)$ yields the change in open-loop disturbance gain with $y_{i}$ uncontrolled, whereas the RDG $\left(=\beta_{i k}\right)$ yields the change in closed-loop disturbance gain with $y_{i}$ under decentralized control (recall the discussion following (29)).

\subsection{Magnitude of inputs needed for rejecting disturbances}

In words the problem we want to solve is the following: "Given specifications on the controlled outputs, $y$, and a set of disturbances, find the smallest possible inputs needed to reject the disturbances".

\subsubsection{Perfect control $(\mathrm{y}=0)$}

Perfect control is possible when we have at least as many inputs as outputs. Mathematically, for simultaneous disturbances the problem to solve is

$$
U_{\min }^{*}=\max _{d}\left(\min _{u}\|u\|\right) \text { s.t. } G u+G_{d} d=0 ;\|d\| \leq 1
$$

Square plants. For square plants the problem $y=0$ has for any given $d$ only one unique solution given by

$$
u=-G^{-1} G_{d} d
$$

Assume that the inputs have been scaled with respect to constraints. Then a frequency dependent plot of the elements of the matrix $G^{-1} G_{d}$ will show for which disturbances and which inputs constraints may be a problem-this will be the case if an element-magnitude is close to 1 or larger.

If we have several disturbances at the same time, then we must find the worst case combination of disturbances. The solution to (35) using the infinity-norm for $d$ and $u$ is given by

$$
U_{\min }^{*}=\|u\|_{\infty}=\left\|G^{-1} G_{d}\right\|_{i \infty}
$$

where the induced infinity-norm of a matrix is equal to its largest row-sum.

It is also instructive to consider the input magnitude using the two-norm. For one disturbance with $d(j \omega)=1$ we have $\|u\|_{2}=\left\|G^{-1} g_{d}\right\|_{2}$, and using (4) we get

$$
\frac{\left\|g_{d}\right\|_{2}}{\bar{\sigma}(G)} \leq\|u\|_{2} \leq \frac{\left\|g_{d}\right\|_{2}}{\underline{\sigma}(G)}
$$

We see that the singular values of the plant provide bounds for the necessary input magnitude, but that the exact value will depend on the direction of the disturbance $\left(g_{a}\right)$. 
Indeed, we may make the lower bound tight by introducing into (36) the disturbance condition number, $\gamma_{d}(G)$, and derive the necessary input magnitude for rejecting a particular disturbance:

$$
U_{\min }^{*}=\|u\|_{2}=\frac{\left\|g_{d}\right\|_{2}}{\bar{\sigma}(G)} \gamma_{d}(G)
$$

More inputs than outputs. In this case there are many possible combinations of inputs that yield $y=0$, and we look for the one with the smallest $\|u\|$. If we use the 2-norm for $u$ and $d$, then Eq. (35) has a simple analytical solution

$$
U_{\min }^{*}=\|u\|_{2}=\bar{\sigma}\left(G^{H}\left(G G^{H}\right)^{-1} G_{d}\right)
$$

\subsubsection{Specified control performance, $\|y\| \leq 1$}

Mathematically, the problem we want to solve is: For the case with one disturbance

$$
U_{\text {min }}=\min _{u}\|u\| \text { s.t. }\|y\|=\left\|G u+g_{d} d\right\| \leq 1 ; d=1
$$

$d=1$ is chosen because it corresponds to the worst case at any frequency. For the case with simultaneous disturbances

$$
U_{\min }=\max _{d}\left(\min _{u}\|u\|\right) \text { s.t. }\left\|G u+G_{d} d\right\| \leq 1 ;\|d\| \leq 1
$$

This problem must be solved numerically. Note that in general all elements in the $u$, $y$, $G$, etc., are complex. We have not specified which norm to use. In general, we would prefer to use the infinity-norm for $d$. For $u$ we prefer to use the infinity-norm when we are concerned about input constraints, and the 2-norm when we are concerned about not generating disturbances for other subprocesses. For $y$ we may also use the infinity-norm or 2-norm.

\subsubsection{Caution}

The above measures (35)-(42) for $\|u\|$ must be used with care when comparing alternative control structures. The reason is that if we are really concerned with the magnitudes of the inputs - either because the inputs are constrained or because they are disturbances to other subprocesses - then we must consider all variables (inputs) that may vary and possibly reach constraints, including inputs that are used for other lower-level control loops which are already closed, or in other ways directly depend on $u$. The "correct" optimization problem when we are concerned about the magnitude of the input signals is then

$$
\hat{U}_{\min }=\min _{u}\|\hat{u}\| ; \hat{u}=\left(\begin{array}{c}
u \\
u_{y}
\end{array}\right)
$$

where $\hat{u}$ is the extended input vector, and $u_{y}=P u$ denotes the other dependent "input" variables (e.g. inputs used in loops that are already closed).

Example. Distillation configurations. Shimizu and Matsubara (1985) use the value of $\|u\|_{2}$ for perfect control to compare the LV-configuration (with $u=[L V]^{T}$ ) and the DV-configuration (with $u=[D V]^{T}$ ). These and other configurations differ only in how the level loops are configured, and for perfect control we get the same values of the five flows, $L, V, D, B$ and $V_{T}$ (i.e. $\|\hat{u}\|$ is the same for all configurations), and a comparison of the input magnitudes based on only a subset (using $\|u\|$ ) is not meaningful.

This does not mean that the "incorrect" measures such as (37), are completely useless when comparing alternative control configurations, but the interpretation in 
terms of the input magnitudes is misleading. Specifically, the comparison makes more sense if we interpret it from a feedback point of view, where a small change in the inputs is desired because it corresponds to a low-bandwidth controller. In this case it is of most interest to consider frequencies corresponding to the closed-loop bandwidth.

\subsection{Output magnitudes in the presence of input limitations}

The problem we want to solve is the following: "Given the requirement $\|u\|<1$ (input constraints) and a set of disturbances, how good control is possible (how small can $y$ be)?". In other words: What is the best closed-loop disturbance sensitivity with input constraints.

Mathematically, for the case with simultaneous disturbances we want to find

$$
Y_{\min }=\max _{d}\left(\min _{u}\|y\|\right) \text { s.t. }\|d\| \leq 1 ;\|u\| \leq 1 ; y=G u+G_{d} d
$$

Again, different norms may be used on both $u$ and $y$, and we usually prefer the infinity-norm on $d$.

\subsection{Maximum disturbance range}

This measure provides for linear plants a generalization of the Resiliency Index (RI) of Morari et al. (1985) to take into account the disturbance model ${ }^{2}, G_{d}$, and extend it to non-zero frequencies. The objective is to find the maximum disturbance range, denoted RI, such that for all

$$
\|d\| \leq R I
$$

we achieve "feasible operation". Here we define "feasible operation" as acceptable performance $(\|y\| \leq 1)$ in spite of limitations on the inputs $(\|u\| \leq 1)$. Mathematically, this problem may be solved by an iterative solution of (44) (with $\|d\| \leq R I$ instead of $\|d\| \leq 1$ ), and adjust RI until $Y_{\min }=1$. Alternatively, we may solve (42) iteratively and adjust RI until $U_{\min }=1$. One may also define the "feasible operation" in terms of perfect control, and compute for this case $R I^{*}(<R I)$.

\section{Example: LV-Distillation}

As an example we consider a distillation column controlled with the LVconfiguration and with three disturbances related to the feed conditions in addition to possible disturbances in $L$ and $V$. We have

$$
y=\left(\begin{array}{c}
\Delta y_{D} \\
\Delta x_{B}
\end{array}\right) ; u=\left(\begin{array}{c}
\Delta L \\
-\Delta V
\end{array}\right) ; d=\left(\begin{array}{c}
\Delta F \\
\Delta z_{F} \\
\Delta q_{F} \\
\Delta L_{d} \\
\Delta V_{d}
\end{array}\right)
$$

The column data are given by Skogestad and Morari (1987a) and scalings have been applied as follows: The outputs have been scaled such that the maximum allowed change in impurity is $100 \%$ (in terms of linearized variables). The allowed input

\footnotetext{
${ }^{2}$ Note that in Morari et al. (1985) $d$ denotes the effect of the disturbances on the outputs, whereas in this paper it denotes a vector of physical disturbances, which affects the output through the disturbance model $G_{d}$.
} 
changes equal the nominal feed rate, i.e. correspond to a $37 \%$ change for $L$ and a $31 \%$ change for $V$. The allowed changes for the disturbances are: 1) A $20 \%$ change in feed rate $F ; 2$ ) a 0.1 mole fraction unit change in feed composition $z_{F} ; 3$ ) a $10 \%$ change in the liquid fraction $q_{F}$; and 4) and 5) the disturbances in the inputs equal $10 \%$ of their allowed changes (i.e. $3.7 \%$ for $L$ and $3.1 \%$ for $V$ ).

Comment: One should be somewhat careful about interpreting the results from this example since they are based on steady-state data only.

We get the following steady-state open-loop model

$$
G=\left(\begin{array}{rr}
87 \cdot 8 & 86 \cdot 4 \\
108 \cdot 2 & 109 \cdot 6
\end{array}\right)
$$

From the gain matrix $G$ we obtain

$$
\begin{gathered}
R G A=\Lambda=G \times\left(G^{-1}\right)^{T}=\left(\begin{array}{rr}
35 \cdot 1 & -34 \cdot 1 \\
-34 \cdot 1 & 35 \cdot 1
\end{array}\right) \\
P R G A=\Gamma=\tilde{G} G^{-1}=\left(\begin{array}{rr}
35 \cdot 1 & -27 \cdot 6 \\
-43 \cdot 2 & 35 \cdot 1
\end{array}\right)
\end{gathered}
$$

A singular value decomposition yields

$$
\bar{\sigma}(G)=197 \cdot 2, \underline{\sigma}(G)=1 \cdot 39, \gamma(G)=141 \cdot 7
$$

1). Open-loop disturbance gain.

$$
G_{d}=\left(\begin{array}{rrrrr}
7.88 & 8.81 & 8.68 & 8.78 & 8.64 \\
11.72 & 11.19 & 10.92 & 10.82 & 10.96
\end{array}\right)
$$

The elements in the matrix $G_{d}$ are larger than 1 so feedback control is needed to reject disturbances. Note that all five disturbances are almost identical when considering their open-loop effects, but we shall see that their directions are quite different, as is apparent when we apply feedback control.

The disturbance condition numbers for the five disturbances are

$$
\gamma_{d}(G)=11.75,1.48,1.09,1.42,1.41
$$

and we note that disturbance 1 is in the most difficult direction, but it is far from being entirely in the "bad" direction which would correspond to a value of $\gamma_{d}(G)$ equal to $\gamma(G)=141 \cdot 7$.

2). The disturbance measures for decentralized control are

$$
\begin{gathered}
C L D G=\Gamma G_{d}=\left(\begin{array}{rrlll}
-47.7 & -0.40 & 2.51 & 8.8 & 0 \\
70.5 & 11.68 & 7.83 & 0 & 11.0
\end{array}\right) \\
R D G=\left(\Gamma G_{d}\right) / G_{d}=\left(\begin{array}{rrlll}
-6.05 & -0.05 & 0.29 & 1.0 & 0 \\
6.01 & 1.04 & 0.72 & 0 & 1.0
\end{array}\right)
\end{gathered}
$$

where / denotes element-by-element division. We note that disturbance 2 has a much smaller effect (only $5 \%$ ) on output 1 than we may expect from the open loop disturbance gains, $G_{d}$, whereas disturbance 1 actually has about six times larger effect.

3). Similar results are obtained when evaluating the partial disturbance gains with the other output perfectly controlled. For example, for output $y_{1}$ uncontrolled and with input $u_{1}$ in manual ( $y_{2}$ constant) the PDG is for the five disturbances $(k=1$ to 5$)$ is

$$
P D G_{11 k}=\left(\begin{array}{lllll}
-1.36 & -0.01 & 0.07 & 0.25 & 0
\end{array}\right)
$$


The combined effect of the disturbances for the four alternative "pairings" is

$$
G_{P D G}=\left\{\sum_{k}\left|P D G_{i j k}\right|\right\}=\left(\begin{array}{ll}
1 \cdot 69 & 2 \cdot 33 \\
2 \cdot 14 & 2 \cdot 87
\end{array}\right)
$$

It is desirable to find "uncontrolled pairings" $u_{j}-y_{i}$ where the $G_{P D G}$-element is less than 1. In our case we see that for $y_{1}$ uncontrolled with $u_{1}$ in manual this is almost satisfied; the worst-case combination for simultaneous disturbances is $\left|y_{1}\right| \leq 1.69$. is

4). The input magnitudes needed for perfect rejection of each disturbance, $d_{k}=1$,

$$
G^{-1} G_{d}=\left(\begin{array}{rrrll}
-0.54 & -0.005 & 0.029 & 0.10 & 0 \\
0.64 & 0.111 & 0.071 & 0 & 0.10
\end{array}\right)
$$

or

$$
\|u\|_{2}=\left(\begin{array}{lllll}
0.837 & 0.111 & 0.076 & 0.10 & 0.10
\end{array}\right)
$$

Again we see that disturbance 1 is the worst. The input needed for rejecting the worst-case combination of disturbances is

$$
U_{\min }^{*}=\|u\|_{\infty}=\left\|G^{-1} G_{d}\right\|_{i \infty}=0.92
$$

and since this value is less than 1 we do not reach input constraints even for the worst-case combination of disturbances. ${ }^{3}$

5). If we find that the inputs needed for perfect control, $U_{\min }^{*}$, are too large (which is almost the case here), then one may consider the inputs needed for obtaining $\|y\|_{\infty}<1$ by solving problems (41) or (42). We use the infinity-norm for $u, d$ and $y$ and obtain $\|u\|_{\infty}$ numerically. For one disturbance at the time (41) yields for the five disturbances

$$
U_{\min }=\|u\|_{\infty}=0.049,0 \cdot 047,0 \cdot 046,0.088,0.046
$$

We note with interest that the input magnitude needed for disturbance no. 1 is now not any larger than for the other disturbances. The reason is probably that with the allowed error for $y$ we need not counteract the part disturbance 1 that acts in the difficult plant direction. For the worst-case combination of disturbances (assuming all elements in the vector $d$ are real) (42) yields $U_{\min }=\|u\|_{\infty}=0.251$. This shows that if we do not require perfect control, but allow an error of 1 in each output ( 0.01 mole fraction units), then we may reduce the input magnitudes by a factor of about four.

6). The best achievable output magnitudes (disturbance sensitivities) in the presence of input constraints, $\|u\|_{\infty} \leq 1$, is obtained by solving problem (44). Since, in our case we already know that we can achieve $y=0$ with $\|u\|_{\infty}=0.92$, the solution is $Y_{\min }=\|y\|=0$ for all disturbances.

7). The maximum disturbance range is obtained by an iterative solution of (41) and (42) until $U_{\min }=1$. We use the infinity-norm for $u$ and $y$. We obtain for each of the individual disturbances using, $d=R I$ in (41)

$$
R_{I}=|d|=2 \cdot 66,16 \cdot 1,20 \cdot 0,17 \cdot 1,17 \cdot 1
$$

Here we find again that disturbance 1 is the most difficult to reject. For simultaneous disturbances using (42) we get $R I=\|d\|_{\infty}=1 \cdot 86$.

\footnotetext{
${ }^{3}$ We here assume that the inputs have been scaled with respect to input constraints. Also, we assume that the other dependent inputs, $D, B$ and $V_{T}$ (cooling), which are used for regulatory control of level and pressure, do not reach constraints (recall discussion following (43)).
} 


\section{Discussion and Conclusion}

The disturbance measures 3, 4, 5 and 6 do not make any assumption about the controller used, and they apply to both feedback and feedforward control. Specifically, note that in (42) and (44) for the case with simultaneous disturbances, we minimize over $u$ in the inner loop. Thus we get the bound on the achievable performance for any control system. Note that these measures do not taking into account that control performance is limited by the presence of RHP-zeros, time delays, model uncertainty, etc. However, these effects may be considered separately, and combined with the results from the disturbance analysis, when performing an overall analysis of controllability.

We have considered six problems related to disturbance sensitivity. Problem 1 simply involves the disturbance transfer matrix $G_{d}$, and frequency-dependent plots may be used to identify the bandwidth requirements for the control system. The solution to problem 2 is the Closed-loop Disturbance Gain (CLDG) introduced by Skogestad and Hovd (1990). The measures resulting from problems 3, 4 and 5 are new. Problems 2 and 3 differ in that the output in consideration is controlled in problem 2, while it is uncontrolled in problem 3. For problems 1 to 3 we find analytical solutions, whereas problems 4, 5 and 6 involve numerical max-min problems.

\section{Appendix. Notation and proofs}

We here consider only a single disturbance at a time and write

$$
y=G u+g_{d} d
$$

where $y$ and $u$ are vectors, $d$ is a scalar, $G$ is square matrix and $g_{d}$ is a vector. (The results may easily be generalized to the case where $d$ is a vector and $G_{d}$ is a matrix.) On element form (scalar notation) (49) becomes

$$
y_{i}=\sum_{l} g_{i l} u_{l}+g_{d i} d
$$

where the scalars $g_{i j}$ and $g_{d i}$ are defined by

$$
g_{i j}=[G]_{i j}=\left(\partial y_{i} / \partial u_{j}\right)_{u_{i \neq j}} ; g_{d i}=\left[g_{d}\right]_{i}=\left(\partial y_{i} / \partial d\right)_{u}
$$

Here the notation $[A]_{i j}=a_{i j}$ denotes the $i j$ 'th element of the matrix $A$, and $[a]_{i}$ the $i$ 'th element of the vector $a$.

Partial derivative notation: Consider a multivariable system with $n+1$ degrees of freedom. In our case there may be $n$ input variables and 1 disturbance. Assume $n$ of these degrees of freedom are kept constant, and collect these in the vector $a$. Then there is one degree of freedom that may be varied, and we denote this variable with the scalar $d$. Now, consider the effect of $d$ on some dependent variable $\alpha$ (also a scalar). This effect is given by

$$
(\partial \alpha / \partial d)_{a}=\left(\frac{\alpha}{d}\right)_{a=0}
$$

where the equality applies since we use deviation variables. In a specific case we may imagine specifying a combination of inputs and outputs, for example, if we set $a$ equal to $\left(y_{i}, u_{l \neq i}\right)$, then this means that the single output $y_{i}$ and the $n-1$ inputs $u_{l \neq i}$ are kept constant (i.e. all $n$ inputs are constant except input $u_{i}$ ). 


\section{Results}

1. Input for perfect control of all outputs:

$$
\left(\partial u_{j} / \partial d\right)_{y}=-\left[G^{-1} g_{d}\right]_{j}
$$

Proof. Set $y=0$ and (49) yields $u=-G^{-1} g_{d} d$ and thus $u_{j}=\left[-G^{-1} g_{d}\right]_{j} d$ and the result follows.

2. Input for perfect control of one output:

$$
\left(\partial u_{j} / \partial d\right)_{y_{j}, u_{l \neq j}}=\left[-(\tilde{G})^{-1} g_{d}\right]_{j}
$$

This result is due to Grosdidier and is presented in Skogestad and Morari (1987a) but without a proof.

Proof. Set $u_{l}=0$ for all $l \neq j$. Then (50) yields

$$
y_{j}=g_{j j} u_{j}+g_{d j} d
$$

and with $y_{j}=0$ we get $u_{j}=-\frac{1}{g_{j j}} g_{d j} d$ and thus

$$
\left(\partial u_{j} / \partial d\right)_{y_{j}, u_{l \neq j}}=-\frac{1}{g_{j j}} g_{d j}=-\left[(\tilde{G})^{-1} g_{d}\right]_{j}
$$

3. Expression for relative disturbance gain (RDG):

$$
\beta_{j} \stackrel{\text { def }}{=} \frac{\left(\partial u_{j} / \partial d\right)_{y}}{\left(\partial u_{j} / \partial d\right)_{y_{j}, u_{l \neq j}}}=\frac{\left[G^{-1} g_{d}\right]_{j}}{\left[(\tilde{G})^{-1} g_{d}\right]_{j}}
$$

Proof. Follows directly from (53) and (54).

4. Partial gain and disturbance sensitivity (PDG) for uncontrolled output with the other outputs perfectly controlled:

$$
\left(\partial y_{i} / \partial u_{j}\right)_{y_{l \neq i}}=\frac{1}{\left[G^{-1}\right]_{j i}}, \quad\left(\partial y_{i} / \partial d\right)_{u_{j}, y_{l \neq i}}=\frac{\left[G^{-1} g_{d}\right]_{j}}{\left[G^{-1}\right]_{\mathrm{ji}}}
$$

Proof. Rewrite (49) as

$$
u=G^{-1} y-G^{-1} g_{d} d
$$

Set $y_{l}=0$ for all $l \neq i$. Then

$$
u_{j}=\left[G^{-1}\right]_{j i} y_{i}-\left[G^{-1} g_{d}\right]_{j} d
$$

and we derive the first result by setting $d=0$ and the second result by setting $u_{j}=0$.

5. The expressions for RGA and RPDG follow by taking ratios of (57) and (51).

REFERENCES

BRISTOL, E. H. (1966). On a new measure of interaction for multivariable process control. IEEE Trans. Autom. Control, AC-11, 133-134.

Hovd, M. and Skogestad, S. (1992). Simple frequency-dependent tools for control system analysis, structure selection and design. Automatica, 28, 989-996.

LUYBEN, W. L. (1988). The concept of "Eigenstructure" in process control. Ind. Eng. Chem. Res., 27, 206-208.

McAvoy, T. J. (1983). Interaction Analysis, ISA Monograph Series, Research Triangle Park, North Carolina, USA. 
MCCune, L. C. and GaLlier, P. W. (1973). Digital simulation: A tool for the analysis and design of distillation controls. ISA Trans., 12, 193-207.

MORARI, M. (1983). Design of resilient process plants III. A general framework for the assessment of dynamic resilience. Chem. Eng. Sci., 38, 1881-1891.

Morari, M., Grimm, W., Oglesby, M. J. and Prosser, I. D. (1985). Design of resilient processing plants VII. Design of energy management system for unstable reactors-new insights. Chem. Eng. Sci., 40, 187-198.

PERKINS, J. D. (1989). Interactions between process design and process control. Preprints IFAC-symposium DYCORD + '89, Maastricht, Netherlands, Aug. 1989, 349-357.

Shimizu, K. and MatSubara, M. (1985). Directions of disturbances and modeling errors on the control quality in distillation systems. Chem. Eng. Commun., 37, 67-91.

SKOGESTAD, S. and Hovd, M. Use of frequency-Dependent RGA for control structure selection. Proc. American Control Conference (ACC), 2133-2139, San Diego, May 1990.

SKOGESTAD, S. and Morari, M. (1987a). The effect of disturbance directions on closed-loop performance. Ind. Eng. Chem. Res., 26, 2029-2035.

SKOGESTAD, S. and MORARI, M. (1987b). Control configuration selection for distillation columns. AIChE Journal, 33, 1620-1635.

Stanley, G., Marino-Galarraga, M. and MCAvoy, T. J. (1985). Short-cut operability analysis I, The relative disturbance gain. Ind. Eng. Chem. Process Des. Dev., 24, 1181-1188.

WAller, K. V., HägGblom, K. E., SANDElin, P. M. and FinNERMAN, D. H. (1988). Disturbance sensitivity of distillation control structures. AIChE Journal, 34, 853-858. 